\section{Repression of factor VIII inhibitor development with apoptotic factor VIII-expressing embryonic stem cells}

\author{
Yoshihiko Sakurai, ${ }^{1,2}$ Shogo Kasuda, ${ }^{3}$ \\ Kohei Tatsumi, ${ }^{1}$ Tomohiro Takeda, ${ }^{1}$ \\ Junko Kato, ${ }^{1}$ Atsushi Kubo, ${ }^{4}$ \\ Midori Shima ${ }^{1}$
}

${ }^{1}$ Departments of Pediatrics, Nara Medical

University School of Medicine, Kashihara;

2Department of Pediatrics, Nara

Prefectural Mimuro Hospital, Sango;

${ }^{3}$ Legal Medicine, Nara Medical University

School of Medicine, Kashihara; ${ }^{4}$ First

Department of Internal Medicine, Nara

Medical University School of Medicine,

Kashihara, Japan

\section{Abstract}

Development of factor VIII (fVIII)-neutralizing antibodies, called inhibitors, is a challenging problem in the management of hemophilia A patients. We explored the possibility of pretreatment with apoptotic fVIII-expressing embryonic stem (ES) cells to prevent the development of fVIII inhibitors. Murine ES cells integrated with the human F8 gene were differentiated into embryoid bodies, dissociated to a single cell suspension, subjected to hypo-osmotic shock to induce apoptosis, and intraperitoneally injected into hemophilia A mice. Inhibitors were induced by periodic intraperitoneal injections of recombinant human fVIII (rhfVIII). In the groups in which intraperitoneal injections of rhfVIII began at 1-3 weeks after pretreatment, the titers of inhibitors were significantly lower after the third administration of rhfVIII compared with that in the control group in which apoptotic Ainv18 ES cells (without the human F8 gene) were used for pretreatment, and continued to show lower levels until the sixth administration of rhfVIII. These results suggest that pretreatment with apoptotic hfVIII-expressing ES cells might be promising for the prevention of fVIII inhibitor development in hemophilia A patients.

\section{Introduction}

Hemophilia A is an X-linked bleeding disorder resulting from an abnormality in the coagulation factor VIII (fVIII) gene. Hemophilia A patients require life-long supplementation with intravenous fVIII. The risk of infectious diseases has dramatically decreased as a result of the development of highly pure plasma-derived and recombinant fVIII. Nonetheless, development of fVIII-neutralizing antibodies, called fVIII inhibitors, remains a challenging problem for the treatment of hemophilia A. Approximately $25-35 \%$ of severe type hemophilia A patients develop inhibitors that reduce or completely negate the benefits of replacement therapy. ${ }^{1,2}$ Furthermore, a major issue is that all gene therapy trials for hemophilia are subject to the risk of developing inhibitory antibodies. ${ }^{3}$ Although immune tolerance induction therapy (ITI) using high amounts of fVIII is effective for eradicating inhibitors, the extremely high cost required for this type of therapy hampers its worldwide application. Moreover, ITI is ineffective in about $30 \%$ of hemophilia A patients with inhibitors. ${ }^{4}$ Thus, it is desirable to develop a novel method to prevent the development of fVIII inhibitors. To this end, several attempts have been made as follows. Madoiwa et al. successfully induced immune tolerance for human fVIII (hfVIII) by intrathymic injection of hfVIII into neonatal hemophilia A mice. ${ }^{5}$ Furthermore, induction of long-term fVIII tolerance can be achieved by administration of lentiviral vectors carrying a canine fVIII transgene to neonatal hemophilia A mice. ${ }^{6}$ Although these approaches were effective, they have several disadvantages such as difficulties in the procedures or the risk of viral vectors causing adverse genetic modification of patients.

The use of apoptotic cells for immune tolerance induction is in line with the current perception that dendritic cells induce peripheral tolerance by capturing cells that normally die during cell turnover. Because apoptotic cells are immunologically silent, ${ }^{7}$ they exert a tolerogenic influence in adaptive immune responses.,8-10 Therefore, a method to prevent antibody production with apoptotic cells has an immunological rationale and is sufficiently promising based on recent studies. ${ }^{11,12}$ However, such an approach has several disadvantages that should be overcome for practical use in the clinical setting of hemophilia A treatment. One of the most concerning issues is the source of fVIII-expressing cells. While the most appropriate cells are autologous cells from an immunological viewpoint, patient-derived cells have a genetically abnormal F8 gene. Thus, ex vivo transfection of the F8 gene into patient-derived fibroblasts has provided a breakthrough for this approach. Su et al. demonstrated that injection of fVIII expression vector-modified apoptotic syngeneic fibroblasts achieves suppression of fVIII inhibitor development in hemophilia A mice. ${ }^{12}$ However, the significant issues in this approach are the complex procedures such as collection of fibroblasts from each patient and transfection of the $F 8$ gene into these cells. Therefore, fVIII-expressing cells derived from histocompatible stem cells [such as embryonic stem (ES) cells or induced pluripotent stem (iPS) cells] will be the most suitable.
Correspondence: Yoshihiko Sakurai, Department of Pediatrics, Nara Prefectural Mimuro Hospital, 1-14-16 Mimuro, Sango, Ikoma-gun, Nara 6360802, Japan.

Tel. +81.745.320505 - Fax: +81.745 .320517 .

E-mail: ysakurai21@gmail.com

Key words: hemophilia, fVIII inhibitors, prevention, embryonic stem cells, apoptosis.

Contributions: YS, conception and design of the study, interpretation of data, and revisions of the manuscript; SK and KT, animal experiments, interpretation of data, literature searches, and preparation of manuscript; TK and JK, cell culture, animal experiments, and collection of data; AK, preparation of ES cells and supervising genetic manipulation; MS supervising study and critical revisions of the manuscript.

Funding: this work was supported by the Mitsubishi Pharma Research Foundation to YS.

Conflict of interests: the authors declare no potential conflict of interests.

Received for publication: 7 December 2012.

Revision received: 10 April 2013.

Accepted for publication: 29 April 2012.

This work is licensed under a Creative Commons Attribution NonCommercial 3.0 License (CC BYNC 3.0).

(c) Copyright Y. Sakurai et al., 2013

Licensee PAGEPress, Italy

Hematology Reports 2013; 5:e9

doi:10.4081/hr.2013.e9

Although we have observed that iPS cells can be differentiated into liver-like populations that show a strong expression level of fVIII mRNA, ${ }^{13}$ fVIII protein expression levels have not yet been examined. Therefore, we attempted to use genetically engineered (hfVIII-transgenic) ES cells as fVIII-expressing stem cells. We explored the possibility of fVIII inhibitor prevention by pretreatment with apoptotic fVIII-secreting ES cells in anticipation of the common and broad usage of iPS cells.

\section{Materials and Methods}

\section{Mice}

fVIII-deficient mice carrying a stop mutation in exon 16 of the $F 8$ gene were generously given to us by Prof. Yoichi Sakata (Research Division of Cell and Molecular Medicine, Jichi Medical University, Shimotsuke, Japan) and used as a model of hemophilia A. ${ }^{14}$ These hemophilic mice were mated with $\mathrm{C} 57 \mathrm{BL} / 6$ mice for at least eight generations to create C57BL/6 fVIII knockout mice. The genotypes of hemophilic mice were 
confirmed by polymerase chain reaction analysis of genomic DNA extracted from peripheral leukocytes. ${ }^{14}$ The mice were maintained as an inbred colony and were between 8 and 12 weeks of age at the beginning of the study. All mice were housed under specific pathogen-free conditions in the animal facility of Nara Medical University Research Center. Blood samples were obtained by orbital plexus bleeding and anticoagulated (9:1) with $0.105 \mathrm{M}$ sodium citrate. Plasma samples were obtained by centrifugation of the blood at $3600 \mathrm{~g}$ for $10 \mathrm{~min}$ at room temperature, divided into aliquots, and frozen at $-80^{\circ} \mathrm{C}$ until analysis. All experiments using mice were approved by the Nara Medical University Animal Use Committee and performed in accordance with the applicable guidelines and regulations.

\section{Induction of factor VIII inhibitors}

Development of fVIII inhibitors in C57BL/6 fVIII knockout mice was induced by weekly intraperitoneal administrations of 4 IU recombinant hfVIII (rhfVIII; Kogenate FS, Bayer Yakuhin, Osaka, Japan) (approximately 100 IU/kg body weight).

\section{Assay for factor VIII inhibitors}

The titers of fVIII inhibitors were assessed by the Bethesda assay using the obtained plasma samples according to a previous report with minor modification. ${ }^{15,16}$ In brief, $50 \mu \mathrm{L}$ of doubling diluted mouse plasma with Owren's Veronal Buffer (more diluted if needed) was incubated with $50 \mu \mathrm{L}$ of normal pooled human plasma at $37^{\circ} \mathrm{C}$ for $2 \mathrm{~h}$. Residual human fVIII activity was measured in a one-stage assay using $50 \mu \mathrm{L}$ of FVIII-deficient human plasma (Sysmex, Kobe, Japan) and a $50 \mu \mathrm{L}$ sample from the previous incubation. Samples were mixed with $100 \mu \mathrm{L}$ of phospholipid activator (APTT-
SLA, Sysmex), incubated at $37^{\circ} \mathrm{C}$ for $3 \mathrm{~min}$, and then mixed with $100 \mu \mathrm{L}$ of $20 \mathrm{mM} / \mathrm{L} \mathrm{CaCl}_{2}$. Clotting times were measured with a coagulometer (KC 10, Amelung, Lemgo, Germany). Coagtrol N (Sysmex) was diluted with Owren's Veronal Buffer to produce a standard curve of fVIII activity. The measurements were made in the linear portion of the response range.

\section{Cell preparations}

Murine Tet-WT-F8 ES cells, in which human F8 gene expression was induced by the tetracycline analog doxycycline, were prepared as described previously. ${ }^{17}$ Briefly, Ainv18 ES cells were transfected with the WT-F8-plox targeting plasmid (wild-type human fVIII gene-loading, lox-targeting plasmid) by electroporation to yield tet-WT-F8, ${ }^{18}$ after which the transfectants were selected with G418 as described previously. ${ }^{19}$ Tet-WT-F8 ES cells were maintained on mouse embryonic fibroblasts as a feeder layer, passaged twice on gelatin-coated dishes, and then induced to form embryoid bodies (EBs) for 6 days. The EBs were stimulated with doxycycline for the last 2 days. Then, EBs were dissociated to a cell suspension by digestion with $0.25 \%$ trypsin/EDTA. As a control, Ainv18 ES cells without the human $F 8$ gene were treated in the same manner.

Apoptosis of the dissociated ES cells was induced according to the osmotic shock method by Liu et al. ${ }^{20}$ Briefly, $1 \times 10^{6}$ cells were washed in RPMI 1640 twice, and resuspended in $1 \mathrm{~mL}$ hypertonic medium ( $0.5 \mathrm{M}$ sucrose, $10 \% \mathrm{wt} / \mathrm{vol}$ polyethylene glycol 1000 , and $10 \mathrm{mM}$ Hepes in RPMI 1640 , pH 7.2) for $10 \mathrm{~min}$ at $37^{\circ} \mathrm{C}$. Then, 10 $\mathrm{mL}$ prewarmed hypotonic medium $\left(40 \% \mathrm{H}_{2} \mathrm{O}\right.$, and $60 \%$ RPMI 1640) was added, and the cells were incubated for an additional $2 \mathrm{~min}$ at $37^{\circ} \mathrm{C}$. Immediately after the incubation, the cells were centrifuged, washed twice with ice-cold phosphate-buffered saline (PBS), and used as apoptotic cells. Induction of apoptosis of the dissociated ES cells was confirmed by flow cytometry analysis with annexin V/7-amino-actinomycin D staining (data not shown).

\section{Administration of apoptotic embry- onic stem cells}

Dissociated and apoptosis-induced Tet-WT-F8 ES cells or control Ainv18 ES cells in $200 \mu \mathrm{L}$ PBS $\left(2 \times 10^{5}\right.$ cells) were intraperitoneally injected into hemophilia A mice. Intraperitoneal injections of rhfVIII for the induction of fVIII inhibitor development were started at 3 days, 1, 2, and 3 weeks after administration of the apoptotic TetWT-F8 ES cells (group D3, W1, W2, and W3, respectively) and after administration of Ainv18 ES cells (control). After the first intraperitoneal injection of rhfVIII, blood sampling was performed just prior to the next intraperitoneal injection of rhfVIII (Figure 1).

\section{Statistical analysis}

Data were presented as the mean \pm standard error of the mean (SEM). Differences between groups were assessed by the Kruskal-Wallis test, followed by Dunn's multiple-comparison post hoc analysis. A P value of less than 0.05 was defined as statistically significant. All statistical analyses were performed using GraphPad Prism (GraphPad Software, San Diego, CA, USA).

\section{Results}

\section{Development of factor VIII inhibitors in hemophilia A mice}

Figure 2 shows the progressive increase of the fVIII inhibitor titer with weekly intraperi-

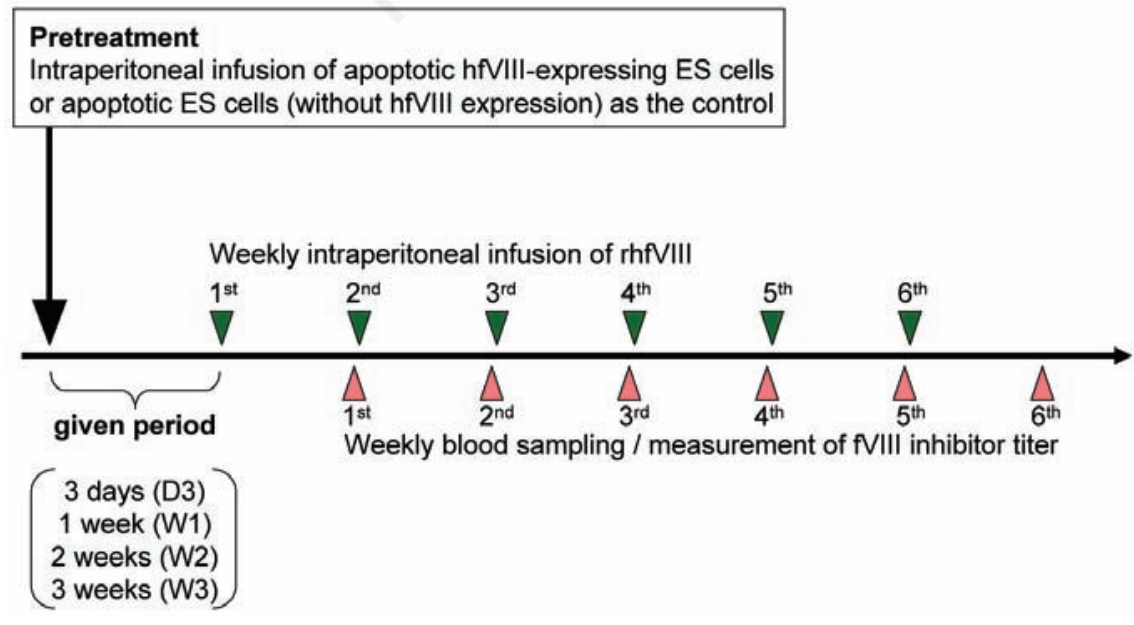

Figure 1. Timeline of the experimental procedure.

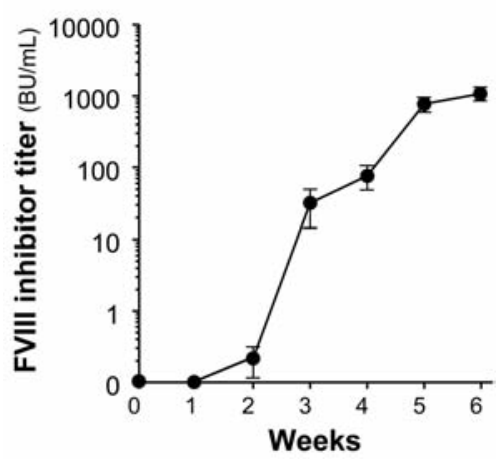

Figure 2. Progressive increase of factor VIII (fVIII) inhibitor titers in hemophilia A mice by weekly intraperitoneal administrations of recombinant human fVIII (rhfVIII) $(n=6-8)$. Inhibitor titers reached a plateau after the fifth administration of rhfVIII. 
toneal administration of rhfVIII to non-pretreated hemophilia A mice $(n=8)$. The fVIII inhibitor titers appeared after the second administration of rhfVIII $(0.22 \pm 0.38 \mathrm{BU} / \mathrm{mL})$. Then, inhibitor titers increased with every following rhfVIII administration and reached $1687 \pm 309 \mathrm{BU} / \mathrm{mL}$ after the six administrations.

\section{Intraperitoneal injection of apoptotic factor VIII-expressing cells attenuates the development of factor VIII inhibitors}

The fVIII inhibitor titers were undetected in both treated and untreated groups before intravenous rhfVIII administration. Those of pretreatment groups at each blood collection point are shown in Figure 3. Pretreatment with apoptotic Ainv18 ES cells (without the human F8 gene), regardless of the period between pretreatment and the first administration of rhfVIII, resulted in no difference in fVIII inhibitor development compared with that in non-pretreatment groups (data not shown). The fVIII inhibitor titers of a group in which intraperitoneal injections of rhfVIII were started at 1 week after administration of Ainv18 ES cells were shown in Figure 3 as a representative control group (Cont).

The fVIII inhibitor titers observed in pretreatment group D3 appeared higher than that in the control group after the second blood sampling but without significance. However, in W1, W2, and W3 groups, the fVIII inhibitor titers were significantly lower than that in the control group at the third blood sampling $(\mathrm{P}<0.05, \mathrm{P}<0.01$, and $\mathrm{P}<0.05$, respectively). The results obtained during the rest of the observation period also showed a similar tendency of lower fVIII inhibitor titers in W1, W2, and W3 groups than those in the control group.

\section{Discussion}

In the current study, we examined the possibility of pretreatment with hfVIII-expressing ES cells for the prevention of fVIII inhibitor development. The efficacy for the reduction of fVIII inhibitor titers depended on the period between pretreatment with apoptotic hfVIIIexpressing ES cells and the first administration of rhfVIII. In the D3 group, pretreatment with apoptotic hfVIII-expressing ES cells resulted in rising inhibitor titers rather than a reduction. However, in groups with a greater than 1 week interval, significant suppressive effects on fVIII inhibitor development were achieved by the pretreatment. These observations suggest that a specified period is required to suppress immune response to fVIII by prior injection of apoptotic hfVIII-expressing ES cells.

The molecular weight of the hfVIII protein is
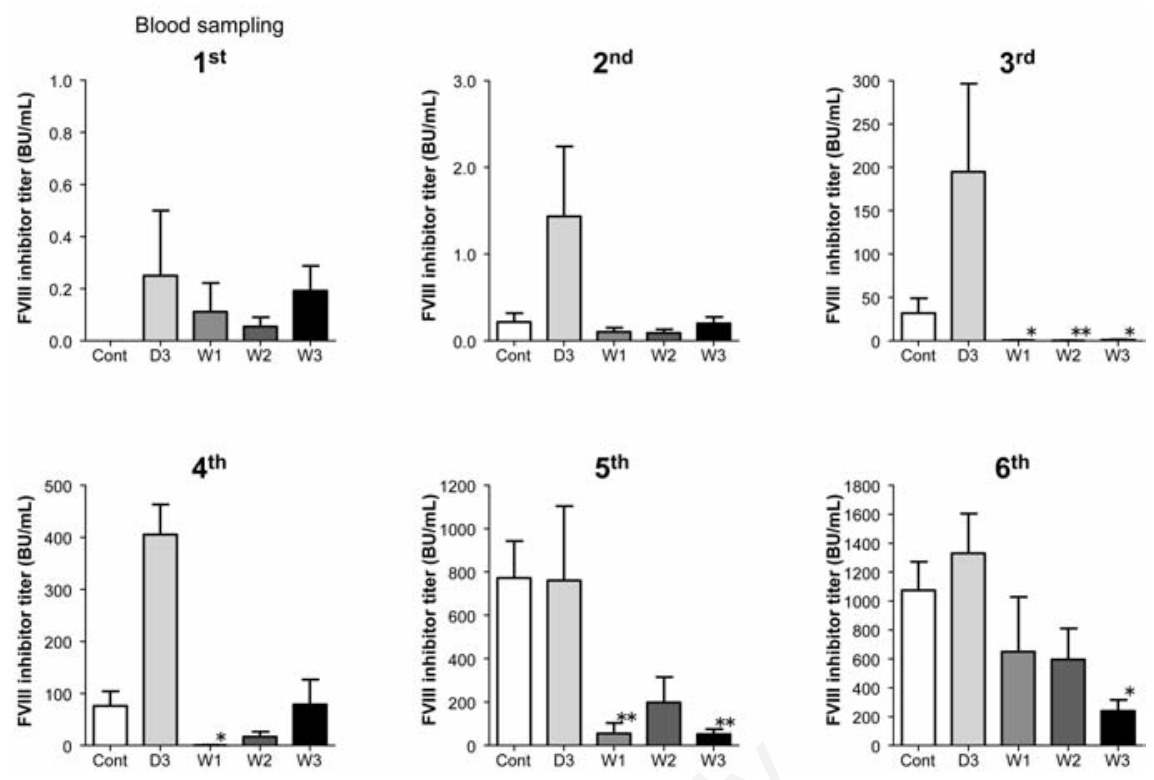

Figure 3. Effects of intraperitoneal injection of apoptotic factor VIII (fVIII)-expressing embryonic stem cells on $\mathrm{fVIII}$ inhibitor development. Intraperitoneal administrations of recombinant human fVIII (rhfVIII) for the induction of fVIII inhibitor development were started at 3 days, 1, 2, and 3 weeks after administration of the apoptotic cells (D3, W1, W2, and W3 groups, respectively) $(n=4-8)$ and 1 week after administration of Ainv18 cells (Cont) $(n=6)$. Data are presented as the mean \pm standard error of the mean. ${ }^{*} P<0.05$, ${ }^{* *} \mathbf{P}<0.01$, compared with the control group.

very large, as much as $280 \mathrm{kDa}$, and multiple epitopes in this protein are recognized by fVIII inhibitors. ${ }^{21}$ Thus, it is a critical issue to clarify whether the fVIII inhibitors that bind multiple epitopes of the fVIII protein can be suppressed by apoptotic hfVIII-expressing cells. Lei et al. found that full-length antigen expressed by B cells can induce immune tolerance for multiple epitopes in a target protein, ${ }^{22}$ because proteolytic cleavage of the protein, selection of the resulting peptides, and peptide presentation are executed by the host's own APCs. Our successful results in the present study support this previous notion. We have no need for identification of the precise peptide epitopes of the fVIII inhibitors or the establishment of ES cells that express each epitope corresponding to a fVIII inhibitor.

The mechanism by which pretreatment with apoptotic hfVIII-expressing ES cells suppresses fVIII inhibitor development remains to be elucidated. Although further investigations are required to address this issue, it might be attributed to the inhibitory effects of the apoptotic ES cells on the proliferation of fVIIIresponsive effector $\mathrm{T}$ cells by antigen-specific $\mathrm{CD} 4{ }^{+} \mathrm{CD} 25^{+}$regulatory $\mathrm{T}$ cells as described by Su et al. ${ }^{12}$

In this study, rhfVIII was injected not intravenously but intraperitoneally. A previous report describes that inhibitor titer elevated to 122.5 BU/mL after five biweekly intravenous injections of $50 \mathrm{U} / \mathrm{kg}$ rhfVIII (total amount of $250 \mathrm{U} / \mathrm{kg}$ ) in hemophilia mouse. ${ }^{5}$ In our study, inhibitor titer did not exceeded $122.5 \mathrm{BU} / \mathrm{mL}$ until total amount of administered rhfVIII reached $500 \mathrm{U} / \mathrm{kg}$, suggesting that our protocol may be inefficient compared to that of the previous report. The difference of administration route as well as applied dose and administration interval may exert influence. Large size of molecules are absorbed slowly through the peritoneal lymphoid system through the stomata of the milky spot. ${ }^{23}$ As intraperitoneally injected fVIII with a molecular size of more than $280 \mathrm{kDa}$ would be therefore absorbed more slowly than intravenously injected fVIII, immune response against rhfVIII might wane. Furthermore, the peritoneal immune system, which contains distinct immune cells such as B1 cells, ${ }^{24}$ may respond to foreign rhfVIII differently from the circulating immune system. Apoptotic ES cells were also administered intraperitoneally. Intraperitoneal administration of apoptotic cells could be applied as intraperitoneal catheters have been used for intraperitoneal chemotherapy. A comparative controlled study of administration routes, intravenous and intraperitoneal should be required to clarify which route is more effective. Furthermore, modification of the protocol for administration of apoptotic fVIII-expressing ES cells, including changes of the administrating cell dose or administration frequency, would be required for complete suppression of fVIII inhibitor development.

Thus, our methodology is not necessarily directly applicable to hemophilia patients with 
inhibitors. Nevertheless, our study opens up a whole new avenue for the prevention of fVIII inhibitor development. Although the ES cells used in this study were genetically engineered (hfVIII transgenic), iPS cell-derived fVIIIsecreting cells will be established in the near future. ${ }^{25}$ In addition, establishment of an iPS cell bank may allow the prevalence of this approach for fVIII inhibitor prevention. ${ }^{26}$

\section{Conclusions}

Our results in this study suggest the effectiveness of pretreatment of apoptotic fVIIIexpressing ES cells on repression of factor VIII inhibitor development. In addition, a specific period might be required to induce immune tolerance by pretreatment with such apoptotic cells. We believe that this approach has potential as a future preventive therapy against fVIII inhibitor development in hemophilia A patients.

\section{References}

1. Kempton CL, White GC 2nd. How we treat a hemophilia A patient with a factor VIII inhibitor. Blood 2009;113:11-7.

2. Hoyer LW. The incidence of factor VIII inhibitors in patients with severe hemophilia A. Adv Exp Med Biol 1995;386:35-45.

3. Gabrovsky V, Calos MP. Factoring nonviral gene therapy into a cure for hemophilia A. Curr Opin Mol Ther 2008;10:464-70.

4. Key NS. Inhibitors in congenital coagulation disorders. Br J Haematol 2004;127: 379-91.

5. Madoiwa S, Yamauchi T, Kobayashi E, et al. Induction of factor VIII-specific unresponsiveness by intrathymic factor VIII injection in murine hemophilia A. J Thromb Haemost 2009;7:811-24.

6. Matsui H, Shibata M, Brown B, et al. A murine model for induction of long-term immunologic tolerance to factor VIII does not require persistent detectable levels of plasma factor VIII and involves contribu- tions from Foxp3+ T regulatory cells. Blood 2009;114:677-85.

7. Kazama H, Ricci JE, Herndon JM, et al. Induction of immunological tolerance by apoptotic cells requires caspase-dependent oxidation of high-mobility group box-1 protein. Immunity 2008;29:21-32.

8. Ferguson TA, Herndon J, Elzey B, et al. Uptake of apoptotic antigen-coupled cells by lymphoid dendritic cells and cross-priming of $\mathrm{CD} 8(+) \mathrm{T}$ cells produce active immune unresponsiveness. J Immunol 2002;168:5589-95.

9. Griffith TS, Kazama H, VanOosten RL, et al. Apoptotic cells induce tolerance by generating helpless $\mathrm{CD} 8+\mathrm{T}$ cells that produce TRAIL. J Immunol 2007;178:2679-87.

10. Sauter B, Albert ML, Francisco L, et al. Consequences of cell death: exposure to necrotic tumor cells, but not primary tissue cells or apoptotic cells, induces the maturation of immunostimulatory dendritic cells. J Exp Med 2000;191:423-34.

11. Gregory CD, Pound JD, Devitt A, et al. Inhibitory effects of persistent apoptotic cells on monoclonal antibody production in vitro: simple removal of non-viable cells improves antibody productivity by hybridoma cells in culture. MAbs 2009;1:370-6.

12. Su RJ, Epp A, Latchman Y, et al. Suppression of FVIII inhibitor formation in hemophilic mice by delivery of transgene modified apoptotic fibroblasts. Mol Ther 2010;18:214-22.

13. Kasuda S, Tatsumi K, Sakurai Y, et al. Expression of coagulation factors from murine induced pluripotent stem cellderived liver cells. Blood Coagul Fibrinolysis 2011;22:271-9.

14. Bi L, Lawler AM, Antonarakis SE, et al. Targeted disruption of the mouse factor VIII gene produces a model of haemophilia A. Nat Genet 1995;10:119-21.

15. Kasper CK, Aledort L, Aronson D, et al. Proceedings: a more uniform measurement of factor VIII inhibitors. Thromb Diath Haemorrh 1975;34:612.

16. Madoiwa S, Yamauchi T, Hakamata Y, et al. Induction of immune tolerance by neonatal intravenous injection of human factor VIII in murine hemophilia A. J Thromb
Haemost 2004;2:754-62.

17. Kasuda S, Kubo A, Sakurai Y, et al. Establishment of embryonic stem cells secreting human factor VIII for cell-based treatment of hemophilia A. J Thromb Haemost 2008;6:1352-9.

18. Kyba M, Perlingeiro RC, Daley GQ. HoxB4 confers definitive lymphoid-myeloid engraftment potential on embryonic stem cell and yolk sac hematopoietic progenitors. Cell 2002;109:29-37.

19. Kubo A, Chen V, Kennedy M, et al. The homeobox gene HEX regulates proliferation and differentiation of hemangioblasts and endothelial cells during ES cell differentiation. Blood 2005;105:4590-7.

20. Liu K, Iyoda T, Saternus M, et al. Immune tolerance after delivery of dying cells to dendritic cells in situ. J Exp Med 2002;196: 1091-7.

21. Sakurai Y, Takeda T, Shima M, et al. The longitudinal alteration of anti-factor VIII alloantibodies in hemophilia A patients treated with immune tolerance induction therapy. In: Pontell E, ed. Immune Tolerance Research Developments. Haup pauge, NY: Nova Science Publishers; 2008. pp 9-26.

22. Lei TC, Scott DW. Induction of tolerance to factor VIII inhibitors by gene therapy with immunodominant $\mathrm{A} 2$ and $\mathrm{C} 2$ domains presented by B cells as Ig fusion proteins. Blood 2005;105:4865-70.

23. Rusznyak I, Foldi M, Szabo G. Absorption of corpuscular particles from serous cavities. In: Youlten L ed. Lymphatics and Lymph Circulation. Oxford, England: Pergamon Press; 1967. pp 478-97.

24. Parra D, Rieger AM, Li J, et al. Pivotal advance: peritoneal cavity B-1 B cells have phagocytic and microbicidal capacities and present phagocytosed antigen to CD4+ T cells. J Leukoc Biol;91:525-36.

25. Xu D, Alipio Z, Fink LM, et al. Phenotypic correction of murine hemophilia A using an iPS cell-based therapy. Proc Natl Acad Sci USA 2009;106:808-13.

26. Belmonte JC, Ellis J, Hochedlinger K, et al. Induced pluripotent stem cells and reprogramming: seeing the science through the hype. Nat Rev Genet 2009;10:878-83. 